\title{
Anaesthetic Management of a Patient with Peripartum Cardiomyopathy: A Case Report
}

\author{
SAHA NC $^{\mathrm{a}}$, ISLAM MS ${ }^{\mathrm{b}}$, AHMED Nc ${ }^{\mathrm{c}}$, ROY SP $^{\mathrm{d}}$, SARKER PK ${ }^{\mathrm{e}}$, JALALUDDIN $^{\mathrm{f}}$
}

\begin{abstract}
Peripartum cardiomyopathy is a rare affliction of healthy, young, females characterized by congestive heart failure, intracardiac thrombus, and frequently death. PPCM is associated with a hypercoagulative state, which can cause thromboembolic complications, may be, the initial demonstration off peripartum cardiomyopathy, which is usually an intracardiac thrombosis. Lower extremity embolism is uncommon. We present a case of $22 \mathrm{yr}$ old female a known case of preeclamtic toxaemia associated with bronchial asthma underwent caesarian section at $36 \mathrm{wks}$ of pregnancy \& developed severe respiratory distress and needed
\end{abstract}

Introduction:

Anaesthetic management in peripartum cardiomyopathy (PPCM) patients can be a challenge to anesthesiologist. ${ }^{1,2}$ The goals of anaesthetic management include maintenance of optimal ventricular preload and after load while avoiding any anaesthesia induced myocardial depression. ${ }^{3}$ A vigilant monitoring both noninvasive and invasive is essential through out the surgery and in the postoperative period to avoid complications like arrhythmias (atrial fibrillation), hypotension, hypoxemia, pulmonary edema, electrolyte disturbances, myocardial ischemia, thromboembolism, and even sudden death. 1,2,3,4,5,6 Acute limb-threatening lower extremity

a. Dr. Nepal Chandra Saha, Consultant, Cardiac Anaesthesiology, Ibn Sina Cardiac Center, Dhaka.

b. Dr. Md. Sirajul Islam, Consultant \& Associate Professor, Department of Cardiac Anaesthesia, Ibrahim Cardiac Hospital \& Research Institute

c. Prof. Dr. Niaz Ahmed, Consultant, Department of Cardio thoracic Anaesthesia \& ICU, Apollo Hospital, Dhaka.

d. Dr. Siba Pada Roy, Associate Consultant, Department of Cardiac Anaesthesia, Ibrahim Cardiac Hospital \& Research Institute.

e. Dr. Pradip Kumar Sarker, Associate Consultant, Department of Cardiac Anaesthesia, Ibrahim Cardiac Hospital \& Research Institute.

f. Prof. Dr. Jalaluddin, Senior Consultant \& Head, Department of Cardiac Surgery, Ibn sina Cardiac Center, Dhaka.

Address of Correspondence: Dr. Md Sirajul Islam..Consultant \& Associate Professor, Department of Cardiac Anaesthesia, Ibrahim Cardiac Hospital \& Research Institute. Mobile No - 01911386975, E-mail: drsislam08@gmail.com,drmukul@dhaka.net

Receive: 25 August, 2013 Accecepted: 22 December, 2014 mechanical ventilation. Two weeks after delivery she developed low back pain with parasthesia of both lower limbs associated with shortness of breath \& diagnosed as a case of infra-renal aortic thrombus extending down to common illiac arteries associated with postpartum dilated cardiomyopathy and admitted at our hospital for embolectomy. Infiltration anaesthesia and conscious sedation with invasive monitoring was planned. Surgery and anaesthesia proceeded uneventfully.

Key Words: Anaesthesia, peripartum cardiomyopathy, thromboembolism, embolectomy.

(Birdem Med J 2015; 5(1): 50-53)

ischemia is uncommon in young adults with no prior symptoms of arterial insufficiency or risk factors for atherosclerotic occlusive disease. Peripartum cardiomyopathy is a rare affliction of healthy, young, females characterized by congestive heart failure, intracardiac thrombus, and frequently death. ${ }^{7}$ PPCM is associated with a hypercoagulative state, which can cause thromboembolic complica-tions, may be, the initial demonstration off peripartum cardiomyopathy, which is usually an intracardiac thrombosis. Lower extremity embolism is uncommon. ${ }^{8,9}$

\section{Case report}

A 22 yr old female with ASA III E, normotensive nondiabetic and known case of preeclamtic toxaemia with bronchial asthma underwent lower uterine caesarian section (LUCS) at 36 wks of pregnancy and delivered a female live baby. After LUCS the patient developed severe respiratory distress which could not be managed with conservative management and needed mechanical ventilation. The arterial blood gas was $\mathrm{P}_{-} \mathrm{H}_{-}$7.414, $\mathrm{PCO}_{2}$ - $36.4 \mathrm{~mm} \mathrm{Hg}, \mathrm{PO}_{2}-49 \mathrm{~mm} \mathrm{Hg}, \mathrm{HCo}_{3}-23.3 \mathrm{mEq} / \mathrm{liter}$, $\mathrm{SpO}_{2}-85.0 \%$, \& A-aDO $272 \mathrm{~mm} \mathrm{Hg}$. The patient was extubuted after three days. Two days after extubation, the patient again developed shortness of breath and was transferred to a cardiac hospital. They treat the patient conservatively as a postpartum dilated cardiomyopathy (DCM) \& gave discharge on request after five days.

Patient again developed low back pain with parasthesia of both lower limbs associated with shortness of breath 
at home. With this complaints patient had been admitted at Apollo hospital and diagnosed as a case of infrarenal aortic thrombus extending down to common illiac arteries. She took discharge on risk bond and admitted at our hospital for embolectomy. Patient was examined thoroughly \& found oriented but anxious. Following parameters were recorded on examination.

- $\quad$ Pulse: 125/ min with regular rhythm;

- $\quad$ blood pressure: 100/70 mm of Hg;

- respiratory rate: 16 breath/min; presence of bilateral basal crepitation ;

- the lower limb was cold, no pulsation and severe excruciating pain;

- $\quad$ ECG revealed sinus tachycardia with $\mathrm{T}$ inversion in $\mathrm{V}_{5} \& \mathrm{~V}_{6}$;

- $\quad$ Bed side echocardiography (ECHO) showed small organized thrombus in apex, severe global hypokynesia, dilated left atrium, left \& right ventricle, mitral \& tricuspid regurgitation ( grade-I).

- Hb-13.5 g/dl, total count of white blood cells-16000/ cmm, neutrophils $80 \%$, platelets-3,17,000/cmm;

- $\quad$ Serum creatinine-3.05 mg/dl, Urea-75mg/dl;

- $\quad$ SGPT-158U/L, SGOT-228U/L;

- Serum sodium”132mmol/L, serum potassium -5.5 $\mathrm{mmol} / \mathrm{L}$, serum $\mathrm{Ca}^{++}-8.2 \mathrm{mg} / \mathrm{dl}$;

- INR-1.37;

- Color Doppler Ultrasound showed Iso to hypoechoic thrombus at infra -renal aorta extending down to common illiac arteries.

On the same day of admission the patient underwent bilateral embolectomy in lower limb at our cardiac surgical operation theatre. Right radial artery and right internal jugular venous cannulation was done for invasive monitoring and medication. In dwelling catheter was insitu. The procedure (Fogarty embolectomy) was done under local anaesthesia and conscious sedation and was maintained with intravenous (i.v) inj. midazolam $1 \mathrm{mg} /$ dose (2 doses), inj. fentanyl 20ìgm/dose (4 doses) \& inj. ketamine 20mg/dose (3 doses). Oxygen $\left(\mathrm{O}_{2}\right)$ inhalation through nasal cannula at a rate of $3 \mathrm{~L} / \mathrm{min}$.

Vertical incision was given over femoral artery (right \& left) on anterior aspect of the thigh. Both femoral arteries were identified \& stabilized with stay suture. Vertical arteriotomy was done \& fogarty catheter introduced beyond the obstruction. Huge amount of clot was extracted from both femoral arteries. After irrigating with heparin solution, femoral arteries were reconstructed with PTFE (polytetrafluoroethylene) conduit on right side and venous patch in the left side.

Total 3.15 hours was required to complete the whole procedure. Through out the procedure the patient was haemodynamically stable with i.v infusion of inj. dopamine 5 ı̀gm $/ \mathrm{kg} / \mathrm{min}$, inj. adrenaline 0.02 Ìgm $/ \mathrm{kg} / \mathrm{min}$ \& ing. GTN 0.2ìgm $/ \mathrm{kg} / \mathrm{min}$. Her heart rate was 120-125 beat/min, blood pressure $-120 / 70 \mathrm{~mm}$ of $\mathrm{Hg}$, Central Venous Pressure (CVP): $7 \mathrm{~cm}$ of $\mathrm{H}_{2} \mathrm{O} \& \mathrm{SpO}_{2}$ : $99 \%$ with satisfactory urine output (50 $\mathrm{ml}$ per hour).

At the end of the procedure (bilateral pedal pulse was established), patient was shifted to intensive care unit (ICU). On first post operative day (POD) she was shifted to Step down ICU, maintaining stable haemodynamic and transferred to general ward on $3^{\text {rd }}$ POD.

\section{Discussion:}

PPCM is characterized with onset of acute congestive heart failure without any demonstrable cause in the last trimester of pregnancy or within the first 6 months after delivery. The incidence of PPCM is reported as 1:300 to 1:15000 in the western literature; however, incidence in Asian subcontinents scenario is lacking due to paucity of data. ${ }^{10}$ The exact etiology of PPCM remains is unknown, but viral, autoimmune, hemodynamic stress of pregnancy, cytokine mediated inflammation, Gqrelated myocyte apoptosis, oxidative stress-induced Cathepsin D production, selenium deficiency, and idiopathic causes are being hypothesized. ${ }^{10,11,12,13}$ Other possible etiologies include nutritional deficiencies, small-vessel coronary artery disease, excessive salt intake, and peripartum fluid shifts ${ }^{14}$. Maternal age more than 30 years, multiparity, eclampsia, obesity, racial origin (African descent), hypertension, malnutrition, and prolonged tocolysis are predisposing factors. ${ }^{10}$ The risk of thromboembolism increases about 5 times during pregnancy, and even higher in postpartum, on account of hypercoagulability, venous stasis, and deliveryinduced vascular injury ${ }^{15,16}$ The differential diagnosis for acute ischaemia of the leg in women after delivery includes an arterio-arterio embolus, thrombosis of a stenotic artery, para-doxical embolization, primary arterial thrombosis due to a hypercoagulable state, and arterial dissection. ${ }^{17}$ Ischaemia threatening a limb is 
extraordinarily rare as the initial presentation of peripartum cardio-myopathy. Cerebral embolism and stroke have been reported as the initial manifestation. ${ }^{18}$

Previous reports of acute ischemia of the leg described women with several months of known ventricular dysfunction prior to a peripheral embolic event. ${ }^{19}$ Another case presented by Paul JG et.al, who was a young, healthy, postpartum female developed acute ischemia of the left leg, and asymptomatic arterial insufficiency of the right lower leg, as the presenting symptoms of peripartum cardiomyopathy, she was anticoagulated with heparin \& managed conservatively. ${ }^{7}$ Most of the literature review done with PPCM \& anaesthesia describing management of patients with familial dilated cardiomyopathy during pregnancy. There are groups of patient who had prenatal diagnosis of PPCM and underwent lower uterine caesarean section (LUCS) under anaesthesia. Anaesthetic management included general anaesthesia, regional anaesthesia, or both ${ }^{(20)}$ Infiltration anaesthesia has also been described in a patient for caesarean section although the patient died eventualy in the postoperative period. ${ }^{21}$

Patients with prenatal diagnosis of PPCM need to be treated by multidisciplinary approach with cardiologists, anaesthetists, and intensivists and neonatologists being actively involved in the obstetric management. Pain and anxiety associated with surgical incision can increase sympathetic nervous system activity with a resultant increase in cardiac output and peripheral vascular resistance leading to an increase in cardiac after load. Effective labor analgesia decreases sympathetic outflow and decreases plasma catecholamine levels. ${ }^{22}$ Multiple modes of anaesthesia have been described in the literature for operative delivery of patients with PPCM. The hemodynamic goals of anaesthesia, however, are common to all approaches. These are to reduce cardiac preload and after load and to prevent any decrease in already compromised cardiac contractility. Any technique or drugs resulting in sudden fall in systemic vascular resistance should be avoided and careful titration of both intravenous and local anaesthetic drugs is important. Wherever possible, it is important to institute invasive monitoring including blood

pressure and central venous pressure before commencement of anaesthesia ${ }^{23,24}$. In general, RA has been used in patients undergoing nonemergent caesarean section with relatively stable hemodynamic, while moderately symptomatic patients or parturient undergoing emergency surgery have received general anaesthesia (GA). ${ }^{21}$

Techniques of RA used include combined spinal epidural (CSE), continuous spinal anaesthesia (CSA), and continuous epidural anaesthesia (CEA). RA provides many advantages for patients with PPCM. Sympathetic blockade associated with RA is beneficial in a decompensated heart as it decreases both preload and after load. ${ }^{20}$ The choice of anaesthetic technique is the anaesthetist's prerogative and if the goals of hemodynamic management are adhered to, the outcome can be expected to be favorable of the anaesthetic technique used. ${ }^{20}$ As we thought that our procedure should be simple that's why we decided to give local anaesthesia \& if needed we should add sedatives in low doses that does not interfere the haemodynamics of this patient.

GA is also preferred in patients with borderline cardiac decompensation as an already dyspneic patient may not be amenable to the procedure of RA. In such a patient, even minor degrees of sympathetic blockade associated with RA may lead to fulminant cardiac failure. Another contraindication to RA is the anticoagulated patient. Excessive reduction of preload may worsen cardiac output, while the decrease in after load can actually jeopardize coronary perfusion in some patients. Opioid based anaesthesia provides good hemodynamic control and obtundation of response to endotracheal intubation but may require postoperative ventilatory support ${ }^{(20)}$.

\section{Conclusion:}

PPCM is a rare disease of unknown cause that affects women in the child bearing years. The diagnosis of PPCM is challenging. Choice of anaesthesia needs to be guided based on the urgency of operation \& severity of PPCM. Early diagnosis, continued monitoring \& prolong therapy may be beneficial in a good amount of patient. Principles of therapy remain almost same as that in heart failure due to any cause, careful selection of drugs is important with due consideration of the patients. The anaesthetist may be involved in the intensive care management of an actually decompensating patient or anaesthetic management. In all the situations, careful \& intense haemdynamic monitoring \& slow \& judicious titration of anaesthetic drug is important to provide good outcome. 


\section{References:}

1. Zangrillo A, Landoni G, Pappalardo F, Oppizzi M, Torri G. Different anesthesiological management in two high risk pregnant women with heart failure undergoing emergency caesarean section. Minerva Anestesiol 2005; 71:227-36.

2. Bhakta P, Biswas BK, Banerjee B. Peripartum cardiomyopathy: Review of the literature. Yonsei Med J 2007; 48:731-47.

3. Heider AL, Kuller JA, and Strauss RA, Wel s SR. Peripartum cardiomyopathya review of the literature. Obstet Gynecol Surv 1999; 54:526-31.

4. Kotekar N, Nagalakshmi NV, Chandrashekar. A rare case of peripartum cardiomyopathy posted for caesarean section. Indian $\mathrm{J}$ Anaesth elective ventilation postoperatively 2007; 51:60-4.

5. McIndoe AK, Hammond EJ, Babington PC. Peripartum cardiomyopathy presenting as a cardiac arrest at induction of anaesthesia for emergency caesarean section. Br J Anaesth 1995; 75:97-101.

6. Bhakta P, Mishra P, Bakshi A, Langer V. Case report and mini literature review: anaesthetic management for severe peripartum cardiomyopathy complicated with preeclampsia using sufetanil in combined spinal epidural anaesthesia. Yonsei Med J 2011;52:1-12.

7. Paul J. Gagne, John B. Newman, Bart E. Muhs. Ischemia due to peripartum cardiomyopathy threatening loss of a leg. Cardiol Young 2003; 13: 209- 211

8. Bennani SL, Loubaris M, Lahlou I, Haddour N, Badidi M, Bouhouch R, Cherti M, Arharbi M Postpartum cardiomyopathy revealed by acute lower limb ischemia. Ann Cardiol Angeiol (Paris). 2003 Dec; 52(6):382-5.

9. Dong-Yeon Kim', Saidul Islam, Nelson Taposh Mondal, Felicity Mussell, and Matthea Rauchholz ${ }^{2}$ Biventricular Thrombi Associated with Peripartum Cardiomyopathy.j health popul nutr 2011 apr;29(2):178-180

10. Bhawna Soni, Gautam P. L., Anaesthetic management of two cases of peripartum cardiomyopathy, Journal of Obstetric Anaesthesia and Critical Care / Jan-Jun 2011 / Vol 1 | Issue 1 pp. 41- 45.

11. Bhakta P, Mishra P, Bakshi A, Langer V. Case report and mini literature review: anaesthetic management for severe peripartum cardiomyopathy complicated with preeclampsia using sufetanil in combined spinal epidural anaesthesia. Yonsei Med J 2011; 52:1-12.

12. Veille JC. Peripartum eardiomyopathies: a review. Am J Obstet Gynecol 1984; 148:805-18.

13. Lampert MB, Hibbard J, Weinert L, Briller J, Lindheimer M, Lang RM. Peripartum heart failure associated with prolonged tocolytic therapy. Am J Obstet Gynecol 1993; 168:493-5.

14. Sanderson JE, Adesanya CO, Anjorin FI, Parry EH. Postpartum cardiac failure - heart failure due to volume overload? Am Heart J 1979; 97, 613-21.

15. ACOG Practice Bulletin No. 84: Prevention of deep vein thrombosis and pulmonary embolism. Obstet Gynecol 2007; 110:429-40.

16. James AH, Abel DE, Brancazio LR. Anticoagulants in pregnancy. Obstet Gynecol Surv 2006; 61(1):59-69.

17. Finley BE. Acute coagulopathy in pregnancy. Med Clin North Am 1989; 73: 723-743.

18. Hodgman MT, Pessin MS, Homans DC, et al. Cerebral embolism as the initial manifestation of peripartum cardiomyopathy. Neurology 1982; 32: 668-671.

19. Bassaw B, Ariyanayagam DC, Roopnarinesingh S. Peripartum Cardiomyopathy and arterial embolism. West Indian Med J 1992; 41: 79.

20. Rashmi Ramachandran, Vimi Rewari, Anjan Trikha Anaesthetic management of patients with peripartum cardiomyopathy,Journal of Obstetric Anaesthesia and Critical Care / Jan-Jun 2011 / Vol 1 | Issue 1,pp,5-12

21. Mellor DJ, Bodenham A. Infiltration anaesthesia in the management of caesarean section in a patient with peripartum cardiomyopathy, 1996; 51:409.

22. Ray P, Murphy GJ, Shutt LE. Recognition and management of maternal cardiac disease in pregnancy. $\mathrm{Br}$ J Anaesth 2004; 93:428-39.

23. Velickovic IA, Leicht $\mathrm{CH}$. Continuous spinal anaesthesia for caesarean section in a parturient with severe recurrent peripartum cardiomyopathy Int J Obstet Anesth 2004;13: 40-3.

24. Pryn A, Bryden F, Reeve W, Young S, Patrick A, McGrady EM. Cardiomyopathy in pregnancy and caesarean section: Four case reports. Int J Obstet Anesth 2007; 16:68-73. 